\title{
Biochemical Analysis of the Bone of SHC Rats with Spontaneous Hypercholesterolemia and Renal Dysfunction
}

\author{
Katsuichi SUDO, Kunio SHIOTA, Tsuneo MASAKI, \\ RYOICHI TSUKUDA AND TAKESHI FUJITA \\ Biology Research Laboratories, Research and Development \\ Division, Takeda Chemical Industries, Ltd., Osaka 532, Japan
}

\begin{abstract}
Spontaneously hypercholesterolemic (SHC) rats exhibit severe abnormalities in renal function and bone metabolism at old ages, in addition to hypercholesterolemia. SHC rats were also found to show endocrine abnormalities such as hyperthyroidism from young ages. In this study, biochemical and microdensitometric analyses were carried out using femurs to characterize further the abnormality in bone metabolism: whether biochemical markers of the bone may be affected by these abnormalities. At 6 weeks of age, the ashed weight and calcium content of the dried femurs were slightly lower in SHC rats than in age-matched Sprague-Dawley (SD) rats. None of the markers of microdensitometric analysis was changed. At 24 weeks of age, the ashed weight of dried femurs and the density of the marrow region of femurs were lower in the SHC rats. The results indicate that SHC rats exhibit severe abnormality in bone metabolism leading to biochemical changes in the bone at old ages whereas changes in bone markers were minimal at young ages before the onset of severe renal dysfunction.
\end{abstract}

Key words: Spontaneously hypercholesterolemic (SHC) rats, Bone, Renal dysfunction.

(Endocrinol Japon 39:147-151, 1992)

SHC RATS, established from SD rats, spontaneously develop focal glomerular sclerosis besides hypercholesterolemia [1, 2, 3]. In 26-weekold SHC rats, marked hypertrophy of the parathyroid gland and severe calcification in soft tissues were noted [3]. The double fluorescence labeling method and contact microradiography demonstrated impaired mineralization of osteoid tissue in the tibial trabecular bone of old SHC rats [4]. The results seemed to indicate that the trabecular bone lesion was characterized by osteitis fibrosa accompanied by osteomalacia [4]. Mild increase in bone resorption in association with endocrine abnormalities such as significant increases in GH, TSH and thyroid hormones was

Received October 21, 1991

Accepted January 17, 1992

Correspondence to: Dr. Katsuichi SUDO, Biology Research Laboratories, Research and Development Division, Takeda Chemical Industries, Ltd., 2-17-85 Jusohonmachi, Yodogawaku, Osaka 532, Japan. also noted in the young SHC rats [5]. The mild increase in bone resorption in the young SHC rats was suggested to be due to the endocrine abnormalities. Thus, SHC rats may serve as a good model for understanding a mutual, causal relationship between these abnormalities giving useful information about knowledge of diseases related with these abnormalities.

In this study, we determined the mineral contents and bone density of femurs, as the endpoints of abnormality in bone metabolism, to confirm and extend previous observations for further clarification of the bone metabolism of SHC rats.

\section{Materials and Methods}

Animals

Male SHC rats bred in Takeda Chemical Ind. 
Ltd., and age-matched SD rats purchased from Charles River, Japan were maintained under the same conditions for at least 2 weeks before the experiments were started. Rats were fed a laboratory chow (CE-2, CLEA Japan) ad libitum. The chow contains $1.25 \mathrm{~g}$ of calcium, $1.06 \mathrm{~g}$ of phosphate, and $200 \mathrm{IU}$ of vitamin $\mathrm{D}_{3}$ per $100 \mathrm{~g}$.

\section{Examination of femurs}

Right femurs stripped of muscle and soft tissues were weighed (wet weight) and kept at $-20^{\circ} \mathrm{C}$ until analyzed by soft $\mathrm{X}$ ray. After the radiological analysis, femurs were washed with $10 \mathrm{ml}$ of water, and delipidated by keeping femurs in $9 \mathrm{ml}$ of $\mathrm{CHCl}_{3}-\mathrm{CH}_{3} \mathrm{OH}(2: 1)$ for 2 days. The delipidation was repeated once more as described above except that femurs were kept in the organic solvent for 3 days. Then, they were dried at $70^{\circ} \mathrm{C}$ for 3 days and weighed (dry weight). The mineral content was determined by weighing ashed femurs after heating the dry bones at $600^{\circ} \mathrm{C}$ for $24 \mathrm{~h}$. The calcium [6] and phosphate [7] contents were determined by dissolving ashed femurs in 7 (for 6-week-old rats) or $10 \mathrm{ml}$ (for 24-week-old rats) of $2 \mathrm{~N} \mathrm{HCl}$ and taking aliquots of the solution.

Femurs were roentgenographed using a soft X-ray machine (Type CSM, Softex Co., Ltd., Japan) and a soft X-ray film (FG, Fuji Film Co., Ltd., Japan). The bone density of femurs in the roentgenograph was measured at points $0.18 / 1$ (distal metaphysis) and 0.43/1 (diaphysis) from the edge of the distal and using the Interactive Image Analyzer System (IBAS II; Carl Zeiss, West Germany) as reported previously [8]. The outer diameter (D) and bone density ( $\sum$ GS/D) in the distal metaphysis; outer diameter (D), inner diameter (d), cortical width $(\mathrm{D}-\mathrm{d})$, cortical thickness index $(\mathrm{CTI})=(\mathrm{D}-\mathrm{d}) / \mathrm{D}$, mean density of both cortical regions $\left(\triangle G S_{\max }\right)=(\triangle G S 1+\triangle G S 2) / 2$, density of marrow region ( $\triangle \mathrm{GS}_{\mathrm{min}}$ ), and bone density ( $\sum \mathrm{GS} / \mathrm{D}$, mean density integrated area/ outer diameter) in the diaphysis were measured following the microdensitometry of Inoue et al. [8].

\section{Statistical Analysis}

Differences between SHC and SD rats were analyzed by $t$-test.

\section{Results and Discussion}

At 6 weeks of age, the SHC and the SD rats did not differ with respect to the wet and dry weights, and the total mineral, calcium, and phosphate contents of femurs (Table 1). However, the mineral and calcium contents of femurs based on the dry weight of the bone were slightly lower with statistical significance in SHC rats.

At 24 weeks of age, the SHC and the SD rats did not differ with respect to the wet and dry weights, and the total mineral, calcium, and phosphate contents of femurs. Based on the wet weight of

Table 1. Mineral contents of femurs of SD and SHC rats at ages of 6 and 24 weeks

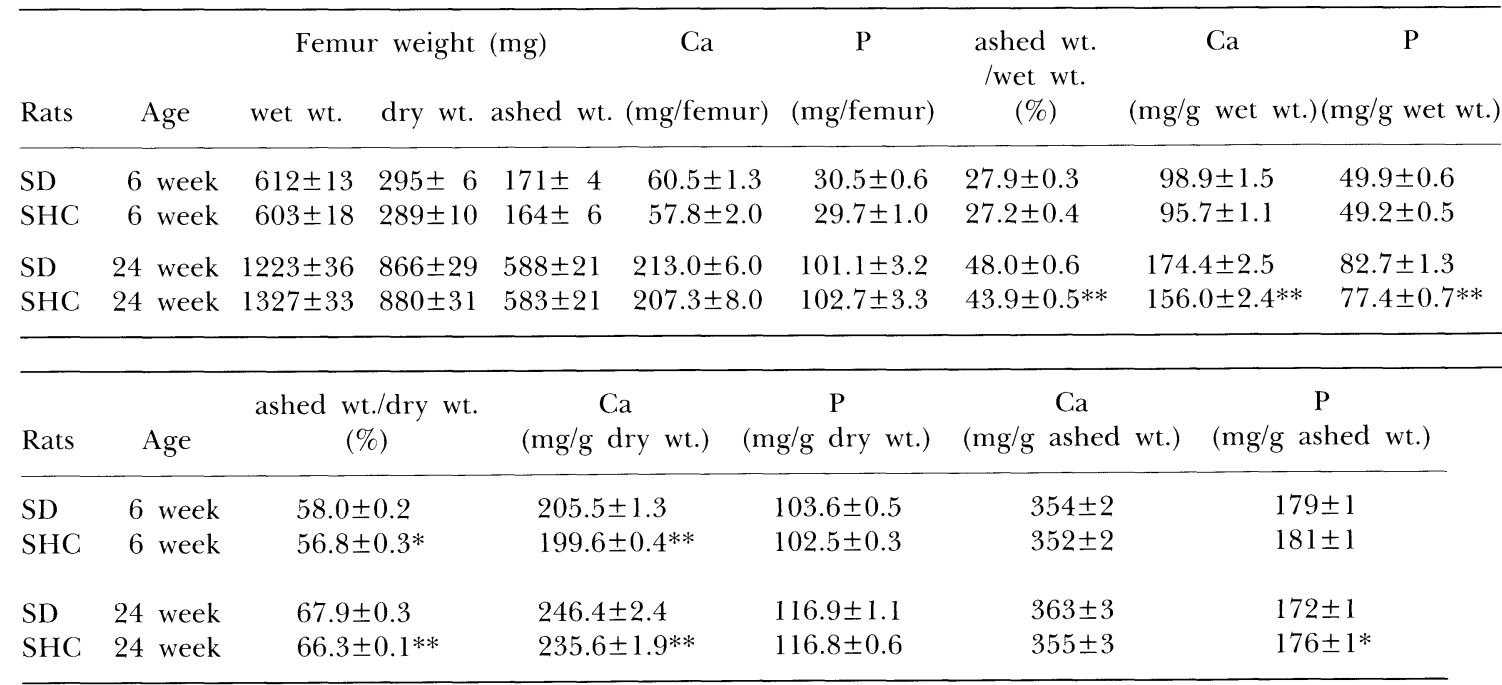

Mean \pm SEM $(\mathrm{n}=6), *, P<0.05 ; * *, P<0.001$ ( $t$-test, compared with SD rats). 


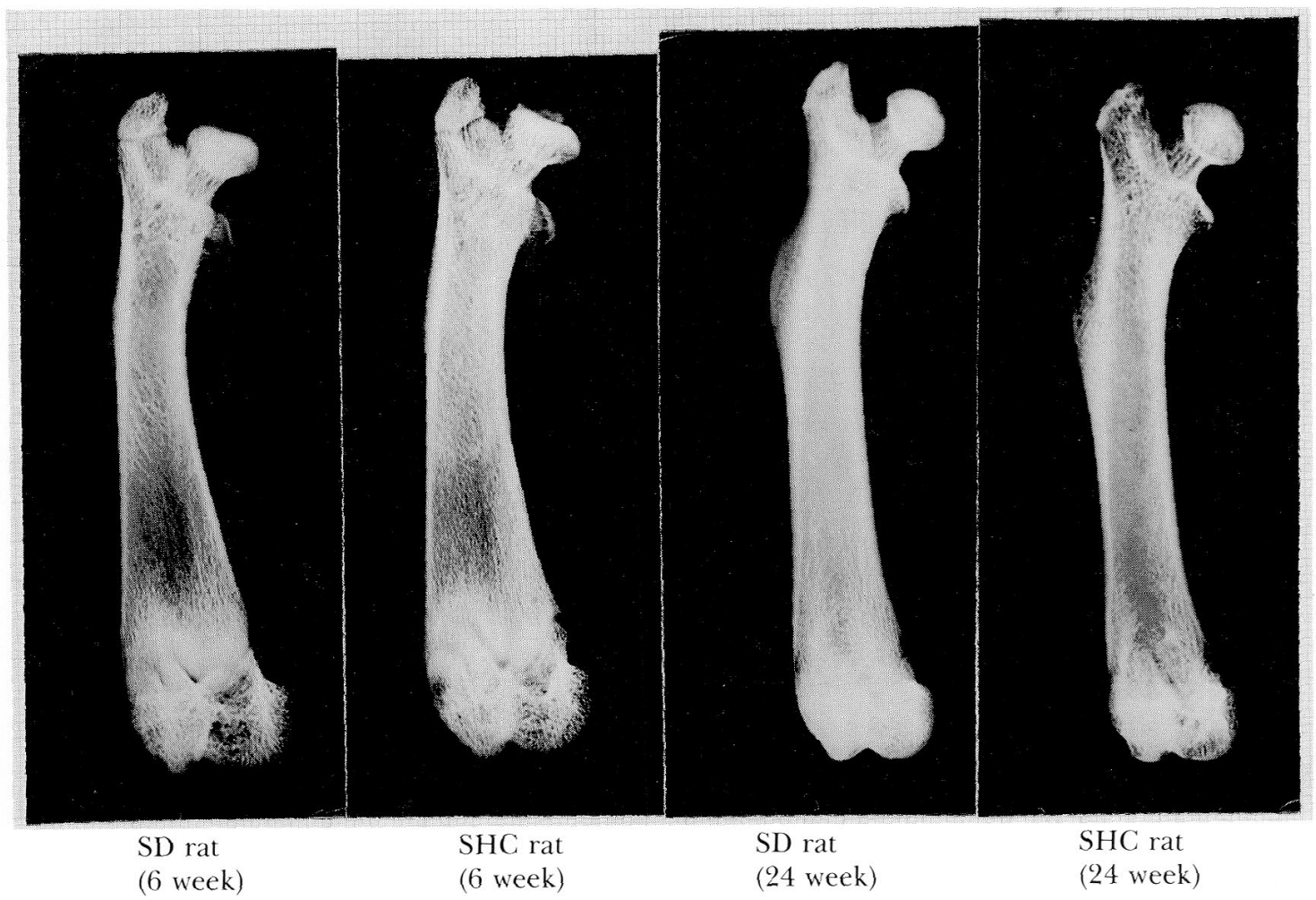

Fig. 1. Roentogenographs of femurs of SD and SHC rats at ages 6 and 24 weeks $(\times 6$ for 6 weeks and $\times 2$ for 24 weeks). At the age of 24 weeks, the bone density in the medullary canal is slightly lower in SHC than in SD rats.

Table 2. Microdensitometric analysis of femurs of SD and SHC rats at ages of 6 and 24 weeks

\begin{tabular}{|c|c|c|c|c|c|c|c|c|c|c|}
\hline \multirow[b]{2}{*}{ Rats } & \multirow[b]{2}{*}{ Age } & \multirow[b]{2}{*}{$\begin{array}{c}\text { Femoral } \\
\text { length } \\
(\mathrm{mm})\end{array}$} & \multicolumn{2}{|c|}{ Metaphysis } & \multicolumn{6}{|c|}{ Diaphysis } \\
\hline & & & $\underset{(\mathrm{mm})}{\mathrm{D}^{\mathrm{a}}}$ & $\Sigma \mathrm{GS} / \mathrm{D}^{\mathrm{b}}$ & $\begin{array}{c}\mathrm{D} \\
(\mathrm{mm})\end{array}$ & $\begin{array}{c}\mathrm{d}^{\mathrm{C}} \\
(\mathrm{mm})\end{array}$ & $\Delta G_{\min }{ }^{d}$ & $\Delta \mathrm{GS}_{\max } \mathrm{e}$ & $\mathrm{CTI}^{\mathrm{f}}$ & $\sum \mathrm{GS} / \mathrm{D}$ \\
\hline SD & 6 week & $28.8 \pm 0.2$ & $5.74 \pm 0.15$ & $0.58 \pm 0.04$ & $3.79 \pm 0.10$ & $3.16 \pm 0.09$ & $0.28 \pm 0.02$ & $1.56 \pm 0.06$ & $0.167 \pm 0.006$ & $0.64 \pm 0.01$ \\
\hline $\mathrm{SHC}$ & 6 week & $28.1 \pm 0.2$ & $5.48 \pm 0.12$ & $0.50 \pm 0.03$ & $3.76 \pm 0.05$ & $3.17 \pm 0.06$ & $0.26 \pm 0.03$ & $1.42 \pm 0.01$ & $0.157 \pm 0.006$ & $0.66 \pm 0.04$ \\
\hline SD & 24 week & $40.8 \pm 0.2$ & $5.88 \pm 0.10$ & $0.81 \pm 0.07$ & $4.42 \pm 0.11$ & $2.97 \pm 0.11$ & $0.93 \pm 0.05$ & $2.02 \pm 0.07$ & $0.328 \pm 0.010$ & $1.14 \pm 0.14$ \\
\hline $\mathrm{SHC}$ & 24 week & $40.6 \pm 0.3$ & $5.88 \pm 0.07$ & $0.73 \pm 0.04$ & $4.63 \pm 0.08$ & $3.21 \pm 0.05$ & $0.72 \pm 0.07 *$ & $2.07 \pm 0.07$ & $0.305 \pm 0.014$ & $1.09 \pm 0.05$ \\
\hline
\end{tabular}

Mean \pm SEM $(n=6) . *, P<0.05$ ( $($-test, compared with SD rats).

a, outer diameter; b, bone density; c, inner diameter; d, density of marrow region; e, mean density of both cortical regions; $f$, cortical thickness index $((\mathrm{D}-\mathrm{d}) / \mathrm{D})$.

femurs, however, the mineral, calcium, and phosphate contents of femurs of the SHC rats were significantly lower. The mineral and calcium contents of femurs on a dry weight-basis were also significantly lower in SHC rats. The phosphate content in ashed bones was also significantly lower in SHC rats at this age.

Microdensitometric analysis of the soft X-ray patterns of femurs showed that none of the morphometric markers of the SHC rats was significantly different from those of the $\mathrm{SD}$ rats at 6 weeks of age (Table 2, Fig. 1).

At 24 weeks of age, the density of the marrow region $\left(\triangle G S_{\min }\right)$ of femurs significantly decreased in the SHC rats. Some of the other morphometric variables, such as $\Sigma \mathrm{GS} / \mathrm{D}$ (bone density) and CTI (cortical thickness index), tended to be slightly lower, and d (inner diameter) tended to be slightly higher, in the SHC at 24 weeks of age. The results are compatible with the previous findings, by 
fluorescent light microscopy and contact microradiography that mineralization defects and increased bone turnover were present in old SHC rats, and confirm and extend these findings [4]. Biochemical markers such as calcium and phosphate contents of the bone may be used as much better markers for bone abnormality than those by histomorphological analyses from the quantitative point of view and from simplicity of assays.

These results indicate that severe abnormality in bone metabolism enough to induce changes in biochemical markers of the bone was observed after onset of severe renal dysfunction which became marked at ages over 12 weeks [3, 4]. Abnormalities in endocrine function such as elevation in serum levels of GH, TSH, $\mathrm{T}_{4}$ and $\mathrm{T}_{3}$ were noted in the SHC rats at the age of 6 weeks [5]. These endocrine abnormalities alone may not be sufficient to induce changes in biochemical markers of the bone. Another possibility is that these abnormalities may compensate each other, balancing bone formation and resorption at young ages, since thyroid hormones and growth hormone act in the opposite directions; the former stimulate bone resorption and turnover [9-12] and the latter enhances 1, 25-hydroxyvitamin $\mathrm{D}_{3}$ formation and vitamin D-dependent calcium binding protein [13-15]. Examination of age-associated changes in vitamin $\mathrm{D}$ metabolism and $\mathrm{PTH}$ secretion in detail would help to characterize the abnormality in bone metabolism of SHC rats. At present, it may be worth mentioning about marked hypertrophy of the parathyroid gland [3] and increased serum level of immunoreactive PTH (human PTH kit for C terminal peptide, EIKEN, Japan; data not shown) in old SHC rats. Thus, hyperthyroidism may be involved in decrease in mineral content of the bone in old SHC rats. Reduction in vitamin D activation due to severe renal dysfunction is also likely related with decrease in ashed weight of the bone.

The abnormality in bone markers at 24 weeks of age is compatible with the quantitative histomorphometric analyses of the tibia of 27-week-old male SHC rats demonstrating the existence of bone changes characterized by osteitis fibrosa accompanied by osteomalacia, changes frequently observed in patients with severe renal dysfunction [16]. Although the exact mechanism of the abnormality in bone metabolism in old SHC rats still remains to be clarified, SHC rats seem to be unique and useful rodents to spontaneously develop abnormalities in endocrine, lipid and bone metabolism as well as renal dysfunction. Therefore, SHC rats may serve as good models for studies on causal relationships between these abnormalities.

\section{Acknowledgments}

We would like to thank Dr. A. Imada, Dr. Y. Imai and Dr. J. R. Miller for their critical advice on the manuscript. We also thank Mr. K. Yoshida and Miss Y, Akinaga for their technical assistance.

\section{References}

1. Imai Y, Matsumura H, Miyajima H, Oka K (1977) Serum and tissue lipids and glomerulonephritis in the spontaneously hypercholesterolemic (SHC) rat, with a note on the effects of gonadectomy. Atherosclerosis 27: 165-178.

2. Matsumura H, Yusa T, Shino A, Imai Y (1985) Development of nephrosis in male SHC rats fed on a diet enriched with protein or fat. $J$ Takeda Research Laboratories 44: 22-29.

3. Tsukuda R, Shino A (1987) Morphological examinations of Spontaneously Hypercholesterolemic (SHC) rat: Spontaneous development of renal lesions and secondary hyperparathyroidism. J Bone Mineral Metab 5: 8-18.

4. Tsukuda R (1987) Histomorphometric study of bone in the spontaneously hypercholesterolemic
(SHC) rat with spontaneously occurring renal failure: An animal model for renal dystrophy. $J$ Bone Mineral Metab 5: 19-29.

5. Shiota K, Takahashi M, Masaki T, Sudo K (1989) Calcium metabolism, serum thyroid-stimulating hormone, prolactin, and growth hormone in spontaneously hypercholesterolemic rats. Proc Soc Exp Biol Med 190: 229-233.

6. Gitelman HJ (1967) An improved automated procedure for the determination of calcium in biological specimens. Anal Biom 18: 521-531.

7. Taussky HH, Shorr E (1953) A microcolorimetric method for the determination of inorganic phosphorus. J Biol Chem 202: 675-685.

8. Inoue T, Kushida K, Miyamoto S, Sumi Y, Orimo H, Yamashita G (1983) Quantitative assessment of 
bone density on X-ray picture. I Japanese Orthop Association 57: 1923-1936.

9. Askenasi R, Demeerster-Mirkinen (1975) Urinary excretion of hydroxylysyl glycosides and thyroid function. J Clin Endocrinol Metab 40: 342-344.

10. Halme J, Vitto J, Kivirikko JI, Saxen L (1972) Effect of triiodothyronine on the metabolism of collagen in cultured embryonic bones. Endocrinology 90: 1476-1482.

11. Mundi GR, Shapiro JG, Canalis EM, Raisz LG (1976) Direct stimulation of bone resorption by thyroid hormones. J Clin Invest 58: 529-534.

12. Kano K, Jones G (1984) Direct in vitro effect of thyroid hormones on 25-hydroxyvitamin $\mathrm{D}_{3}$ metabolism in the perfused rat kidney. Endocrinology 114: 330-336.

13. Spanos ED, Barett D, MacIntyre I, Pike JW,
Safilian EF, Haussler MR (1978) Effect of growth hormone on vitamin D metabolism. Nature 273: 246-247.

14. Wongsurat N, Armbrecht HJ, Zensner TV, Forte LR, Davis BB (1984) Effect of hypophysectomy and growth hormone treatment on renal hydroxylation of 25 hydroxycholecalciferol in rats. $J$ Endocrinology 113: 1387-1392.

15. Elizabeth M, Bruns H, Vollmer SS, Bruns DE, Overpeck JG (1983) Human growth hormone increases intestinal vitamin D-dependent calciumbinding protein in hypophysectomized rats. Endocrinology 113: 1387-1392.

16. Malluche H, Faugere M-C (1990) Renal bone disease 1990: An unmet challenge for the nephrologist. Kidney International 38: 193-211. 